\title{
A Note on Gradient Solitons in Three-Dimensional Riemannian Manifolds
}

\author{
Fatemah Mofarreh and Uday Chand De*
}

(Dedicated to the memory of Prof. Dr. Aurel BEJANCU (1946 - 2020))

\begin{abstract}
We charecterize three-dimensional Riemannian manifolds endowed with a special type of vector field if the Riemannian metrices are gradient Yamabe solitons and gradient Einstein solitons respectively.
\end{abstract}

Keywords: Gradient Yamabe soliton, gradient Einstein soliton, Riemannian manifold.

AMS Subject Classification (2020): Primary: 53C15 ; Secondary: 53C25.

\section{Introduction}

There was a problem for a compact Riemannian manifold $(M, g)$ of $\operatorname{dim} n \geq 3$ to find a metric conformal to $g$ such that the scalar curvature of the new metric is constant. In 1960, H. Yamabe [19] claimed that he had found a solution of the problem. Eight years later, Trudinger [17] found some errors to this solution. However, the problem was named 'Yamabe problem' in keeping with his name. In 1988, R. S. Hamilton [9] introduced the notion of Yamabe flow. Yamabe flow is an intrinsic geometric flow and this is the process to deform the metric of the Riemannian manifold, and which satisfies

$$
\frac{\partial}{\partial t} g=-r g, \quad g(0)=g_{0},
$$

where $r(t)$ is the scalar curvature of the metric $g(t)$. Yamabe soliton metrices stay self-similar under the Yamabe flow for noncompact manifold.

According to Hamilton, a Riemannian metric $g$ of an n-dimensional complete Riemannian manifold $(M, g)$ is said to be a Yamabe soliton if it satisfies

$$
£_{X} g=(r-\lambda) g,
$$

for a real number $\lambda$ and a smooth vector field $X$, where $r$ is the scalar curvature of $g$ and $£$ denotes the Liederivative operator. The vector field is called soliton field of the Yamabe soliton. A Yamabe soliton is said to be shrinking, steady or expanding according to $\lambda>0, \lambda=0$ or $\lambda<0$, respectively.

In this matter, Deshmukh and Chen [8] briefly studied Yamabe soliton to find adequate conditions on the soliton vector field $X$ so that the metric of the Yamabe soliton is of constant scalar curature. For further studies one can see ([6], [18]).

If $X=D f$ for some smooth function $f$ where $D$ is the gradient operator of $g$, then (1.1) takes the form

$$
\nabla^{2} f=(r-\lambda) g
$$

where $\nabla^{2}$ denotes the Hessian of $g$. So gradient Yamabe soliton is a special type of Yamabe soliton. The function $f$ is called potential function, and if $f$ is constant, then the gradient Yamabe soliton is said to be trivial.

To understand the existence of gradient Yamabe solitons one can see the paper [4]. Also, Gradient Yamabe 
solitons have been investigated by several authors such as ([1], [2], [3], [7], [11], [15]) and many others. In [10], Hsu proved that for dimension $n \geq 3$, the metric of any compact Yamabe gradient soliton $\left(M^{n}, g\right)$ is of constant scalar curvature.

The notion of gradient Einstein soliton on a Riemannian manifold was introduced by Catino and Mazzieri [5], which is defined as follows:

If $(M, g)$ be a Riemannian manifold satisfying

$$
S(X, Y)-\frac{1}{2} r g(X, Y)+\nabla^{2} f=\lambda g,
$$

for some function $f: M \rightarrow \mathbb{R}$ and some constant $\lambda$, then the metric $g$ is called gradient Einstein soliton. Gradient Einstein soliton is said to be trivial when $f$ is conatant. Catino et al. [5] proved that all compact gradient Einstein soliton is trivial. So, removing the term compactness our interest on investigation gradient Einstein solitons on Riemannian three manifolds.

Motivated by the above works, in the present paper we characterize three dimensional Riemannian manifolds admitting gradient Yamabe solitons and gradient Einstein solitons. Precisely we prove the following Theorems:

Theorem 1.1. If the metric of a three dimesional Riemannian manifold $M^{3}$ endowed with a special type of vector field is a gradient Yamabe soliton, then the soliton is trivial. Moreover, the manifold is locally isometric to the unit sphere $S^{3}(1)$.

Theorem 1.2. If the metric of a three dimensional Riemannian manifold $M^{3}$ endowed with a special type of vector field is a gradient Einstein soliton, then the manifold is of constant curvature.

\section{Preliminaries}

In this section we shall recall some fundamental results on Riemannian manifolds. Let $\left(M^{n}, g\right)$ be a Riemannian manifold of dimension n. In $\left(M^{n}, g\right)$ the Riemann curvature tensor, Ricci tensor and scalar curvature are denoted by $R, S$ and $r$ respectively. It is known that Riemannian metrics have interesting properties. In particular in a three dimensional Riemannian manifold $\left(M^{3}, g\right)$ the Riemannian curvature tensor has a special form which is given by

$$
\begin{aligned}
R(X, Y) Z & =S(Y, Z) X-S(X, Z) Y+g(Y, Z) L X-g(X, Z) L Y+ \\
& -\frac{r}{2}[g(Y, Z) X-g(X, Z) Y],
\end{aligned}
$$

$L$ being the Ricci operator defined by $g(L X, Y)=S(X, Y)$. Due to this expression of the curavature tensor in $\left(M^{3}, g\right)$ the Weyl curvature tensor vanishes. Several authors have characterized $\left(M^{3}, g\right)$ with various structures such as contact structure and complex structure in ([12], [13], [14]) and many others.

It may be mentioned that every compact, orientable three-dimensional manifold $M$ has a contact structure [16], i.e., there exist a global 1-form $A$ such that $A \wedge d A \neq 0$ everywhere.

Throughout the paper we assume that the three dimensional Riemannian manifold $M^{3}$ admits a unit vector field $V$ such that

$$
\nabla_{X} V=X+A(X) V
$$

where $A$ is a 1-form associated to the vector field $V$ such that

$$
A(X)=g(X, V),
$$

for any vector field $X$ in $M^{3}$ and $\nabla$ is the Levi-civita connection with respect to $g$.

From (2.2) it can be easily seen that

$$
\begin{gathered}
R(X, Y) V=A(Y) X-A(X) Y, \\
\left(\nabla_{X} A\right) Y=g(X, Y)+A(X) A(Y),
\end{gathered}
$$




$$
S(X, V)=2 A(X) .
$$

If we substitute $Y=Z=V$ in (2.1) and using (2.4) and (2.6), then we infer

$$
L X=\left(\frac{r}{2}-1\right) X+\left(3-\frac{r}{2}\right) A(X) V .
$$

This implies

$$
S(X, Y)=\left(\frac{r}{2}-1\right) g(X, Y)+\left(3-\frac{r}{2}\right) A(X) A(Y) .
$$

\section{Proof of our main results}

Before providing the detail proof of our main Theorems, we need the following:

Lemma 3.1. For a three-dimensional Riemannian manifold, we have

$$
V r=6(6-r) .
$$

Proof: Taking covarient derivative of (2.7) with respect to an arbitrary vector field $Y$ we have

$$
\begin{aligned}
\left(\nabla_{Y} L\right) X & =\frac{Y r}{2} X-\frac{Y r}{2} A(X) V+\left(3-\frac{r}{2}\right)[g(X, Y)+A(X) A(Y)] V \\
& +\left(3-\frac{r}{2}\right) A(X)[Y+A(Y) V] .
\end{aligned}
$$

Operating inner product with an arbitrary vector field $Z$ in the above equation, we obtain

$$
\begin{aligned}
g\left(\left(\nabla_{Y} L\right) X, Z\right) & =\frac{Y r}{2} g(X, Z)-\frac{Y r}{2} A(X) A(Z)+\left(3-\frac{r}{2}\right)[g(X, Y)+A(X) A(Y)] A(Z) \\
& +\left(3-\frac{r}{2}\right) A(X)[g(Y, Z)+A(Y) A(Z)],
\end{aligned}
$$

and substituting $Y=Z=e_{i}, 1 \leq i \leq 3$, where $\left\{e_{i}\right\}$ is an orthonormal basis for the tangent space of $M$ and keeping in mind the well known result div $L=\frac{1}{2} g r a d r$, the foregoing relation reduces to

$$
\operatorname{Vr} A(X)=6(6-r) A(X),
$$

which easily follows (3.1). This completes the proof.

Proof of Theorem 1.1: The gradient Yamabe soliton equation (1.2) can be written as

$$
\nabla_{X} D f=(r-\lambda) X
$$

Taking covariant derivative of the above equation along an arbitrary vector field $Y$ we have

$$
\nabla_{Y} \nabla_{X} D f=(Y r) X+(r-\lambda) \nabla_{Y} X .
$$

Applying the foregoing equation repeatedly and (3.5) in the well known expression $R(X, Y) Z=\nabla_{X} \nabla_{Y} Z-$ $\nabla_{Y} \nabla_{X} Z-\nabla_{[X, Y]} Z$ we obtain

$$
R(X, Y) D f=(X r) Y-(Y r) X .
$$

Contracing $X$ in the above equation yields $S(Y, D f)=-2 Y r$, and relating this with (2.8) gives

$$
\left(\frac{r}{2}-1\right) Y f+\left(3-\frac{r}{2}\right)(V f) A(Y)+2 Y r=0 .
$$

For $Y=V$ the foregoing equation provides

$$
V r=-V f
$$


Taking inner product with the vector field $V$ in (3.6) and making use of (2.4) we can easily obtain

$$
(Y f) A(X)-(X f) A(Y)=(X r) A(Y)-(Y r) A(X) .
$$

Replacing $Y=V$ in the above equation we have

$$
X f=-X r .
$$

Making use of (3.8) and (3.9) in the Eq. (3.7) and keeping in mind $V r=6(6-r)$ we can find

$$
\left(3-\frac{r}{2}\right)[Y r-6(6-r) A(Y)]=0 .
$$

This implies either (i) $r=6$ or (ii) $Y r=6(6-r) A(Y)$.

Case (i): If $r=6$, then from (3.9) we can write $X f=0$, i.e., $f=$ constant. Also, using $r=6$ in (2.8) we reveal that

$$
S(X, Y)=2 g(X, Y)
$$

Again using the above equation in (2.1) we get

$$
R(X, Y) Z=g(Y, Z) X-g(X, Z) Y .
$$

Hence the manifold $M^{3}$ is of constant curvature 1. This means that the manifold $M^{3}$ is locally isometric to the unit sphere $S^{3}(1)$.

Case (ii): If $Y r=6(6-r) A(Y)$, and since $X f=-X r$, we have

$$
D f=-6(6-r) V .
$$

Taking covarient derivative with respect to $V$ and using (2.2) infer that

$$
\nabla_{V} D f=24(6-r) V \text {. }
$$

On the other hand, the Eq. (3.5) provides

$$
\nabla_{V} D f=(r-\lambda) V
$$

and this equation together with (3.12) we can conclude $r=\frac{1}{25}(\lambda+144)$. Hence, from (3.9) we obtain $f=$ contant.

This completes the proof.

Proof of Theorem 1.2: The equation (1.3) of gradient Einstein soliton becomes

$$
\nabla_{X} D f=-Q X+\frac{1}{2} r X+\lambda X
$$

By remembering the expression of curvature tensor $R(X, Y)=\left[\nabla_{X}, \nabla_{Y}\right]-\nabla_{[X, Y]}$, the above equation provides

$$
R(X, Y) D f=\left(\nabla_{Y} Q\right) X-\left(\nabla_{X} Q\right) Y+\frac{1}{2}(X r) Y-\frac{1}{2}(Y r) X .
$$

Contracing $X$ in the foregoing equation we have

$$
S(Y, D f)=-\frac{1}{2} Y r
$$

and relating this relation with (2.6) we have

$$
V f=-\frac{1}{4} V r .
$$


Now, keeping in mind the relation (3.2), the Eq. (3.14) can be rewritten as

$$
R(X, Y) D f=\frac{X r}{2} A(Y) V-\frac{Y r}{2} A(X) V+\left(3-\frac{r}{2}\right)[A(X) Y-A(Y) X],
$$

and taking inner product with the vector field $V$ in the foregoing equation we get

$$
g(R(X, Y) D f, V)=\frac{X r}{2} A(Y)-\frac{Y r}{2} A(X) .
$$

On the other side, (2.4) gives

$$
g(R(X, Y) V, D f)=A(Y) X f-A(X) Y f .
$$

For $X=V$, the previous two equations yields

$$
\left(\frac{V r}{2}+V f\right) A(Y)=\frac{Y r}{2}+Y f .
$$

Now, if we use $V f=-\frac{1}{4} V r$, the above equation is transformed to

$$
\frac{Y r}{2}=\frac{3}{2}(6-r) A(Y)-Y f .
$$

By setting $X=D f$ in (2.8) and connecting with (3.15) we have

$$
\left(\frac{r}{2}-1\right) Y f+\left(3-\frac{r}{2}\right) A(Y)(V f)+\frac{1}{2} Y r=0 .
$$

Using (3.16) and (3.18) in the foregoing equation yields

$$
\left(\frac{r}{2}-2\right)\left[Y f+\frac{3}{2}(6-r) A(Y)\right]=0 .
$$

Hence, either $r=4$ or $Y f=\frac{3}{2}(r-6) A(Y)$.

Case (i): If $r=4$, then $V r=0$, so $V f=0$. Hence from (3.17) we get $f=$ constant, and using this in (3.13) we can conclude that the manifold becomes an Einstein manifold.

Case (ii): If $Y f=\frac{3}{2}(r-6) A(Y)$, then $D f=\frac{3}{2}(r-6) V$. This implies

$$
\nabla_{V} D f=6(6-r) V
$$

and by setting $X=V$ in (3.13) we have

$$
\nabla_{V} D f=\left(\frac{r}{2}+\lambda-2\right) V
$$

Equating (3.20) and (3.21) we obtain $r=\frac{76-2 \lambda}{13}=$ constant. Using this in (3.17) we can conclude $f=$ constant. Hence the manifold becomes an Einstein manifold. Being three dimensional the manifold is a manifold of constant curvature.

This finishes the proof.

\section{References}

[1] Blaga, A. M.: On gradient eta-Einstein solitons. Kragujevac Journal of Mathematics. 42, 229-237 (2018).

[2] Blaga, A. M.: Some geometrical aspects of Einstein, Ricci and Yamabe solitons. J. Geom. Sym. Physics. 52, 17-26 (2019).

[3] Blaga, A. M. and Ozgur, C.: Almost eta-Ricci and almost eta-Yamabe solitons with torse-forming potential vector field. Quaestiones Mathematicae. https://doi.org/10.2989/16073606.2020.1850538

[4] Cao, H. D., Sun, X. F. and Zhang, Y. Y.: On the structure of gradient Yamabe solitons. Mathematical Research Letters. 19, 737-774 (2002).

[5] Catino, G., Mazzieri, L.: Gradient Einstein soliton. Nonlinear Anal., 132, 66-94 (2016).

[6] Chen, B.Y. and Deshmukh S.: Yamabe and quasi-Yamabe solitons on Euclidean submanifolds. Mediterr. J. Math. 15, 194 (2018).

[7] De, U.C., Chaubey, S.K. and Suh, Y.J.: A note on almost co-Kahler manifolds. Int. J. of Geo. Methods in Modern Phy. 17 (10), 2050153 (2020). 
[8] Desmukh, S. and Chen, B. Y.: A note on Yamabe solitons. Balk. J. Geo. Appl. 23, 37-43 (2018).

[9] Hamilton, R.S.: The Ricci flow on surfaces. Mathematics and general Relativity, Contemp. Math. 71, 237-262(1998).

[10] Hsu, S.Y.: A note on compact gradient Yamabe solitons. J. Math. Ana. Appl. 388, 725-726 (2012).

[11] Karaca, F.: Gradient Yamabe solitons on Multiply Warped product Manifolds. Int. Electronic J. Geom. 12, 157-168 (2019).

[12] Karatsobanis, J. N. and Xenos, P. J.: On the new class of contact metric 3-manifolds. J. Geom. 80, 136-153 (2004).

[13] Koufogiorgos, T.: On a class of contact Riemannian 3-manifolds. Results in Math. 27, 51-62 (1995).

[14] Kowalski, O.: An explicit classification of 3-dimensional Riemannian spaces satisfying $R(X, Y) . R=0$. Czechoslovak Math. J. 46(121), 427-474 (1996).

[15] Suh, Y.J. and De, U.C.: Yamabe solitons and gradient Yamabe solitons on three-dimensional $N(k)$-contact manifolds. Int. J. Geom. Methods Mod. Phys. 17 (12) 2050177, 10 pp. (2020).

[16] Thurston, W. P. and Winkelnkemper, H. E.: On the existence of contact forms. Proc. Amer. Math. Soc. 52, 345-347 (1975).

[17] Trudinger, N. S.: Remarks concerning the conformal deformation of Riemannian structures on compact manifolds. Annali della Scuola Normale Superiore di Pisa - Classe di Scienze, Serie 3. 22, 265-274 (1968).

[18] Wang, Y.: Yamabe soliton on 3-dimensional Kenmotsu manifolds. Bull. Belg. Math. Soc. Simon Stevin. 23, 345-355 (2016).

[19] Yamabe, H.: On a deformation of Riemannian structures on compact manifolds. Osaka Math. J. 12 21-37 (1960).

\section{Affiliations}

\section{FATEMAH MOFARREH}

AdDress: Mathematical Science Department, Faculty of Science, Princess Nourah Bint Abdulrahman University, Riyadh 11546, Saudi Arabia.

E-MAIL: fyalmofarrah@pnu.edu.sa

ORCID ID:0000-0002-2116-7382

\section{UDAY CHAND DE}

AdDREss: Department of Pure Mathematics, University of Calcutta, 35, Ballygunge Circular Road, Kol700019, West Bengal, India.

E-MAIL: Uc_de@yahoo.com

ORCID ID:0000-0002-8990-4609 